\title{
Measurement of Uterine Leiomyomata Volume Pre and Post Uterine Artery Embolization Aided by MRI
}

Tamara M. Abdullah, Samer M. Botros, Wafaa R. Aly

Department of Radiodiagnosis, Faculty of Medicine, Ain Shams University

Corresponding author: Tamara M Abdullah, Mobile: 01011569494; Email: dr.tamara2017@ gmail.com

\begin{abstract}
Background: uterine fibroid embolization (UFE) is now evolving to be the first-line treatment for symptomatic uterine leiomyomata alongside the conventional surgical treatment of hysterectomy and myomectomy. Cochrane review comprises six RCTs comparing UAE versus hysterectomy or myomectomy for management of symptomatic leiomyomata where UFE had similar short and midterm outcomes, inclusive of symptomatic relief, quality of life and patient satisfaction, while benefiting from uterine preservation, shorter hospital stay and a faster recovery before resuming ordinary life activities. These benefits are in line with the minimally invasive nature of the embolization procedures. Aim of the Work: $t$ this study aimed to review and illustrate the role of MR imaging in the pre- and post-procedural assessment for uterine fibroid patients undergoing uterine artery embolization. Patients and Methods: the current study was carried out in Radiodiagnosis Department, Faculty of Medicine, Ain shams University and specialized private radiology centers during the period between April 2017 and December 2017. It included 30 patients that were referred from gynecology clinic with their ultrasonographic reports to Radiodiagnosis Department, body imaging unit with a view to carry-out pelvic MRI with contrast. Results: imaging follow-up via pelvic MRI with contrast was obtained in 30 patients at 3, 6 months after treatment. The mean uterine volume was reduced by $25 \%$ (standard deviation was SD 0.03763) 3 months after treatment and was further reduced by a mean of 30\% (SD 0.02638 ) by six months after treatment. Post-procedural subtraction MRI revealed mean myoma enhancement to be $5.6+0.5 \mathrm{SI}$ in contrast to $133.4+18.3$ SI prior to UFE. The mean dominant fibroid volume reduced by $34 \%$ (SD 0.09247) after 3 months, $44 \%$ (SD 0.05994) after 6 months. A cumulative reduction in both uterine and dominant leiomyoma mean volume from baseline to 6 months post - UAE was evident with significant interval changes between 3, 6 months follow up ( $\mathrm{p}$ 0.000) Conclusion: the MRI findings following UAE vary with the interval from embolization and success of the procedure. MRI with its multiplanar capabilities was typically employed to evaluate the uterus following UAE for fibroid infarction, size reduction, location change, persistent enhancement, fibroid recurrence, changes in adenomyosis, and unexpected complications that may require surgical intervention or identify women who would benefit from repeated UAE. Recommendations: further studies on a larger scale of patients are needed to confirm the results obtained by this work.
\end{abstract}

Keywords: uterine leiomyomata volume, uterine artery embolization, MRI.

\section{INTRODUCTION}

Management of symptomatic uterine leiomyomata traditionally encompasses diverse treatments ranging from medical treatment up to invasive hysterectomy ${ }^{(1)}$. Symptomatic fibroids used to be treated with hysterectomy whenever having unsatisfactory outcome with medical treatments. Hysterectomy is an invasive procedure that is associated with significant morbidities, 10$15 \%$ complication rates as well as lengthy hospital stay and prolonged recovery time ${ }^{(2)}$.

Patient's desire to preserve their uterus has prompted the development of uterus sparing alternatives as laparoscopic, hysteroscopic myomectomies ${ }^{(3)}$. Presence of multiple fibroids with variable sizes, locations, adding to associated pain, high post-operative morbidity as well as symptoms recurrence ( $25 \%$ of patients] have made myomectomy not feasible in all circumstances ${ }^{(4)}$. Uterine artery embolization (UAE) is a minimally invasive treatment alternative for management of symptomatic myomas ${ }^{(5)}$. It was first reported as a potential treatment option in 1995 and has since been proven to be an efficacious and safe method with a relatively high success rate in minimizing pain, bulk symptoms and bleeding linked to uterine fibroids. UAE is increasingly being preferred over surgical hysterectomy or myomectomy due to reduced morbidity, shorter hospitalization, and relative uterine preservation when compared to surgical resolution ${ }^{(6)}$.

Leiomyomas are hyper vascular benign lesions with large vessels furnishing higher vascular flow relative to the normal myometrial vasculature. This permits preferential targeting and occlusion of end arterial branches that perfuse leiomyomas during the delivery of the particulate embolization agent used during UAE. Embolization of these end arterial branches ultimately results in infarction, coagulative necrosis, and eventual shrinkage of the targeted leiomyomas ${ }^{(7)}$. Owing to higher accuracy in assessing the size, number and location of uterine leiomyomas, Magnetic resonance imaging (MRI) is the preferable method over transvaginal 
US in the pre- and post-procedural assessment for $\mathrm{UAE}^{\left({ }^{(8)}\right.}$. Moreover, MRI is more sensitive than US in diagnosing and excluding further causes of pelvic pain such as adenomyosis or endometriosis.

Evaluation of vascular supply and collateral vasculature, that may be advantageous in pretreatment planning, can be confidently performed with MR techniques. Moreover, MR imaging can be utilized in anticipating treatment response, failure and leiomyoma recurrence, as well as evaluating treatment complications ${ }^{(9)}$.

\section{AIM OF THE WORK}

This study aimed to review and illustrate the role of MR imaging in the pre- and postprocedural assessment for uterine fibroid patients undergoing uterine artery embolization.

\section{PATIENTS and METHODS}

During the period between April 2017 and December 2017, our study was carried out in Radiodiagnosis Department, Faculty of Medicine, Ain shams University and specialized private radiology centers. It included 30 patients that were referred from gynecology clinic with their ultrasonographic reports to radiodiagnosis department, body imaging unit with a view to carry-out pelvic MRI with contrast. The study was approved by the Ethics Board of Ain Shams University.

\section{A. Inclusion criteria}

Our study was conducted upon 30 female patients who were suffered from uterine leiomyoma(s). All women were first assessed clinically for their most annoying symptom/s; the symptoms were grouped as follows:

(1) 22 patients having menstrual irregularities as heavy menstrual bleeding nominated as bleeding associated with prolongation in time and / or increased amount of the usual menstrual periods relative to the patient's prior experience or resulting in severe anemia.

(2) 5 patients with pressure symptoms related to mass effect of the leiomyoma.

(3) 2 patients presented with infertility attributable to the myoma (no other definable cause for infertility) and refusing surgical intervention.

(4) 1 patient suffering from pelvic pain and heaviness.

\section{B. Exclusion criteria}

The exclusion criteria comprised patients who are pregnant; whom presented with pedunculated sub mucosal or cervical leiomyomas; those with renal dysfunction (High serum creatinine $>2 \mathrm{mg} / \mathrm{dl}$ ), patients previously had life threatening contrast allergy, claustrophobia and those having metallic implants, cardiac pace maker, aneurysmal clipping.

\section{Technique of pelvic MRI}

Patients were offered UFE as primary line of management where pelvic MRI with post contrast study was utilized preoperatively to evaluate the volume of dominant uterine leiomyoma/s, determine their location, and exclude other co-existent pathologies as well as to assess their vascularity which gives a clue about treatment feasibility however non-avidly enhancing tumors weren't excluded in this study.

Prior to MRI, patients were subjected to:

1- Proper history taking, full clinical assessment

2- Pre-procedural assessment of renal function inclusive of blood urea \& serum Creatinine

3- Administration of an ante-cubital intravenous catheter

4- Detailed explanation of imaging procedure with obtaining with written informed consent.

Images were acquired for all cases using 1.5/3 Tesla magnetic field MRI with highperformance gradient systems.

- Patient position: the patient lies in a supine position (Head first).

- Coil: phased array body coil

Pre- and post-procedural MR evaluation was performed utilizing the identical protocol. Axial T1-weighted gradient echo in-phase and opposed-phase sequences, axial, sagittal and coronal fast spin-echo T2-weighted sequences, and axial, sagittal, and coronal T1-weighted fatsuppressed two- or three-dimensional pre- and post-contrast imaging is obtained.

- T1-weighted images provide an anatomic overview of the pelvis [In and out of phase] allowing for further characterization of incidental pelvic abnormalities such as ovarian dermoids, which may lose signal on the opposed-phase sequences relative to the inphase sequences due to microscopic fat content. Non-enhanced T1-weighted fatsuppressed imaging is useful in identifying blood products, which can be seen with hemorrhagic degeneration of leiomyomas or with adenomyosis or endometriosis.

- T2-weighted sequence delineates the zonal anatomy of the uterus where the outer most myometrium is intermediate signal, the junctional zone is hypo intense and the innermost endometrial strip is hyper intense. It also allows for accurate localization of the leiomyomas whether submucosal, intramural or subserous. intracavitary submucosal myomas were excluded for fear of expulsion with its sequale. 
- Contrast-enhanced imaging is essential in determining the vascularity and viability of the targeted leiomyomas where they were characterized as non-enhancing or enhancing by subjective comparison of the leiomyoma to the myometrium on post-contrast images

- MR angiography of the pelvic vasculature may be useful in accurately mapping the pelvic vasculature.

Following MRI, UFE procedures were done under local anesthesia with fluoroscopic control using monoplane Toshiba unit with a $5 \mathrm{~F}$ sheath (Radifocus, TERUMO) and a $5 \mathrm{~F} \mathrm{Cobra}^{2}$ catheter (Cordis) with a $0.35 \mathrm{~F}$ hydrophilic guide wire (Radifocus; TERUMO).

Bilateral selective catheterization of uterine arteries till its transverse portion was implemented via bilateral femoral puncture followed by simultaneous embolization utilizing embosphere particles (Biosphere Medical) 500- $700 \mu \mathrm{m}$ (43.3\% of patients), $700-900 \mu \mathrm{m}$ ( $23.3 \%$ of patients) up to 900-1200 $\mu \mathrm{m}(13.3 \%$ of patients) followed by torpedoes of gel foam in 6 patients in order to achieve complete flow cessation. Unilateral UAE embolization was performed in one patient due to technical difficulties as a result of problematic arterial spasm.

The technical end point was stasis in the main uterine artery for not less than 10 cardiac beats. Post-procedural pain was controlled with analgesics and non-steroidal anti-inflammatory agents.

\section{Image Interpretation and procedural outcome}

Post- procedure outcome was evaluated in multiple aspects; technical, physical, psychological and clinical improvement. As a tool to assess technical success of UAE; all patients were subjected to pelvic MRI with contrast six months following the procedure to evaluate reduction in both uterine, dominant leiomyoma volumes as well as need for re-interventions whether repeated UAE or subsequent myomectomies and hysterectomies. The volumes of the uterus and the dominant leiomyomata were calculated in $\mathrm{cm}^{3}$ pre-procedural as a base line and 6 months post-procedural using the formula for a simplified prolate ellipse ( $\mathrm{L} \mathrm{X} \mathrm{W}$ X D X .542) as described by Orsini et al. ${ }^{(\mathbf{1 0})}$.

The uterine fibroid shrinkage volume inferred from pre and post-operative MRI scans will be correlated with symptomatic improvement/ deterioration or stability. On clinical basis, followup of the patien'ts symptoms was monitored three and six months after discharge by verbal questionnaire. The bleeding, pressure and pain symptoms improvements were graded as follow: (Significantly worse, 1 - Slightly worse; 2 -
Stationary; 3 - Slightly improved; 4 - Significantly improved; 5). Patient psychological satisfaction at these follow-ups visits was also rated as: Satisfied (3), neither (2), dissatisfied (1).

To estimate the physical progress, patient's data as regard length of hospital stay, days till return to normal activity, reported complications were assembled. According to Society of Interventional Radiology (SIR) standards; complications were subdivided into: 1- Major complication that necessitates longer hospital stay than expected or that leaves long-lasting adverse effect. 2- Minor complications that can be self limiting or solved on a day case admission.

Descriptive data obtained were the procedure parameters, uterine and leiomyoma volume changes, and questionnaire responses. As baseline parameters, the patient's age, most annoying symptom, the uterine and dominant fibroid volumes, sum of leiomyomata, and site of dominant leiomyoma were all gathered.

\section{Statistical analysis}

IBM SPSS statistics (V. 22.0, IBM Corp., USA, 2013) was used for data analysis. Data were expressed as Median and Percentiles for quantitative non-parametric measures and both number and percentage for categorized data.

The following tests were done: 1Comparison between 2 dependent groups for parametric data using Paired t test. 2- Chi-square test to study the association between each 2 variables or comparison between 2 independent groups as regards the categorized data. The probability of error at 0.05 was considered sig., while at 0.01 and 0.001 are highly sig. Multiple linear regression was used to determine if leiomyoma enhancement, myomatous volume, location (intramural, subserosal, submucosal), and patient age were independent predictors of fibroid shrinkage after UAE.

\section{RESULTS}

\section{Demographics}

A total of 30 cases were gained from target population and the results from 30 patients were analyzed.

\section{Presenting symptoms}

A total of 30 females with average age of 36.5 (28-43 year) came presenting with uterine leiomyomata where heavy menstrual bleeding was the most common complaint represented by $73 \%$ (22 patients) in our study. The $2^{\text {nd }}$ most common clinical presentation was pressure symptoms as urinary frequency and constipation which accounts for $17 \%$ (5 patients). Apart from 3\% (1 patient) presented with intolerable pelvic pain; the 
remaining 7\% (2 patients) came seeking for pregnancy (Fig. 1).

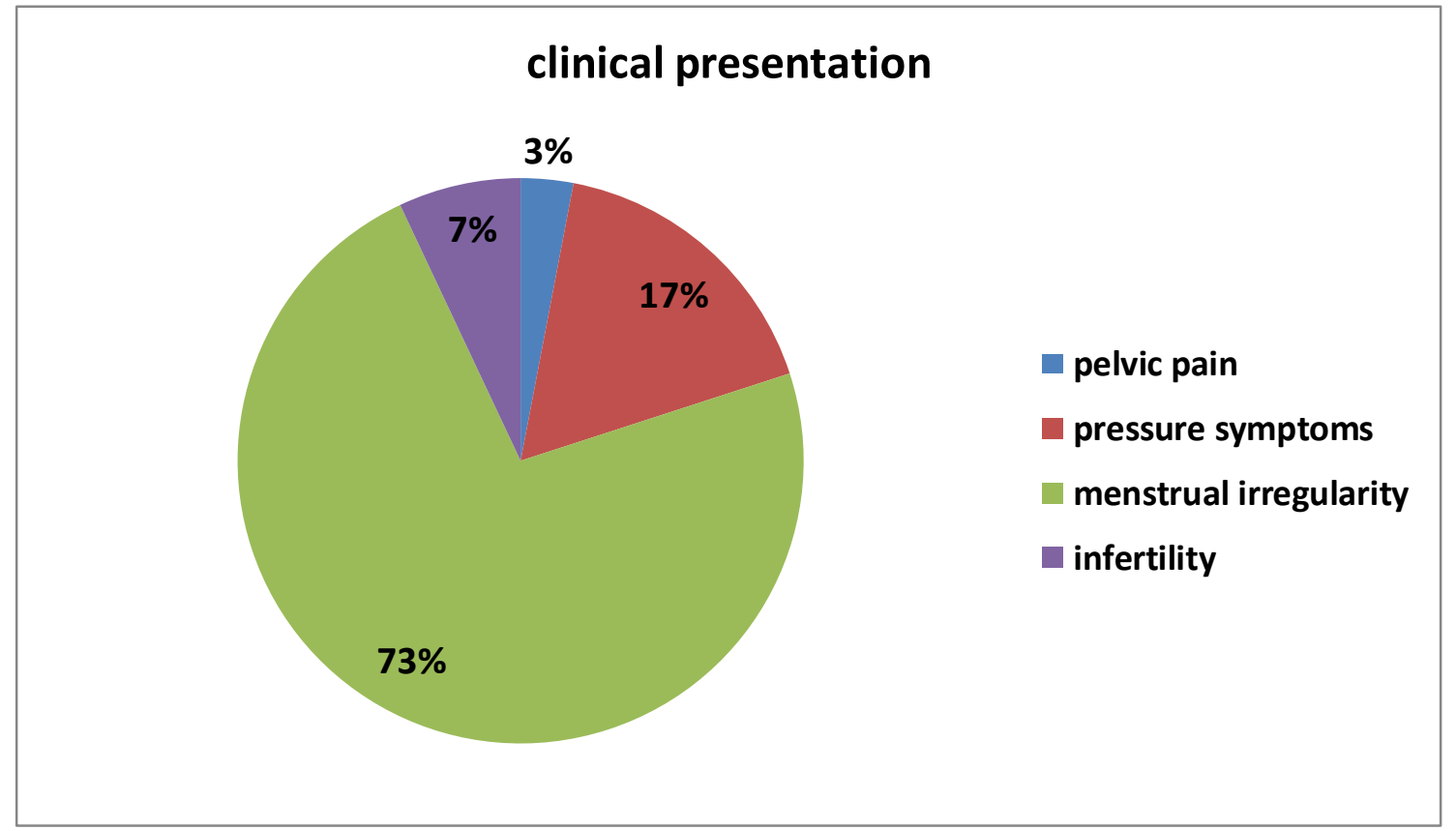

Fig. 1: patients categorized according to clinical presentation

Patients were informed of various conservative and interventional treatment approaches where UFE was picked out as a primary line of management.

Preprocedural MRI revealed an average number of 4 (range: 1-13) leiomyomas per patient. Five patients $(16.6 \%)$ present with only 1 leiomyoma whereas $56.7 \%$ of patients $(n=17)$ harbor $2-6$ myomas. More than 6 leiomyomas were seen in $26.7 \%$ of patients $(n=8)$ (Fig. 2). With reference to myomas location in relation to uterine layers: $23.3 \%(n=7)$ were submucosal where the majority $60 \%(n=18)$ was intramural and the minority $16.7 \%(n=5)$ belonged to sub-serosal myomas (Fig. 3).

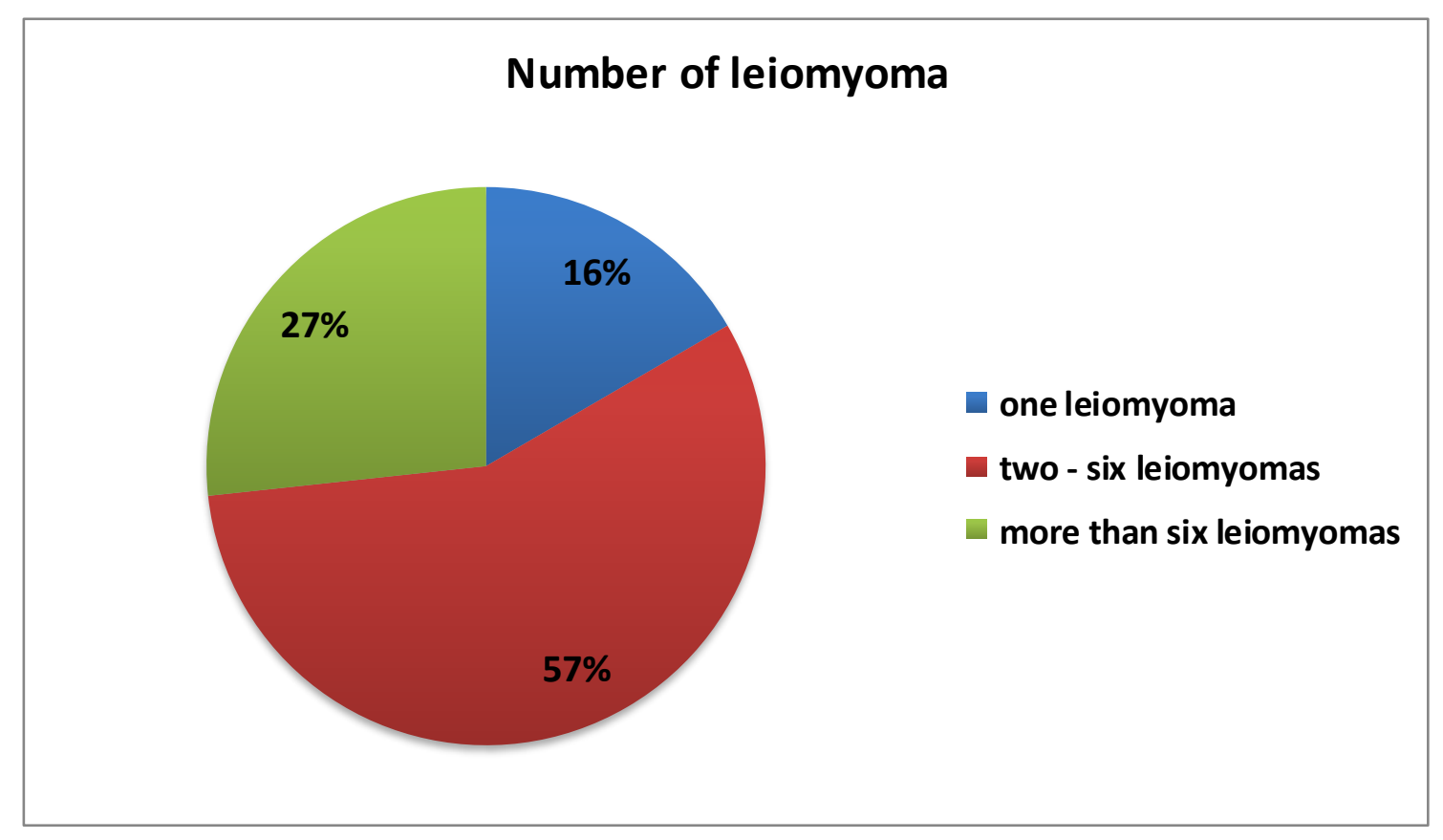

Fig. 2: number of leiomyomas in our study 


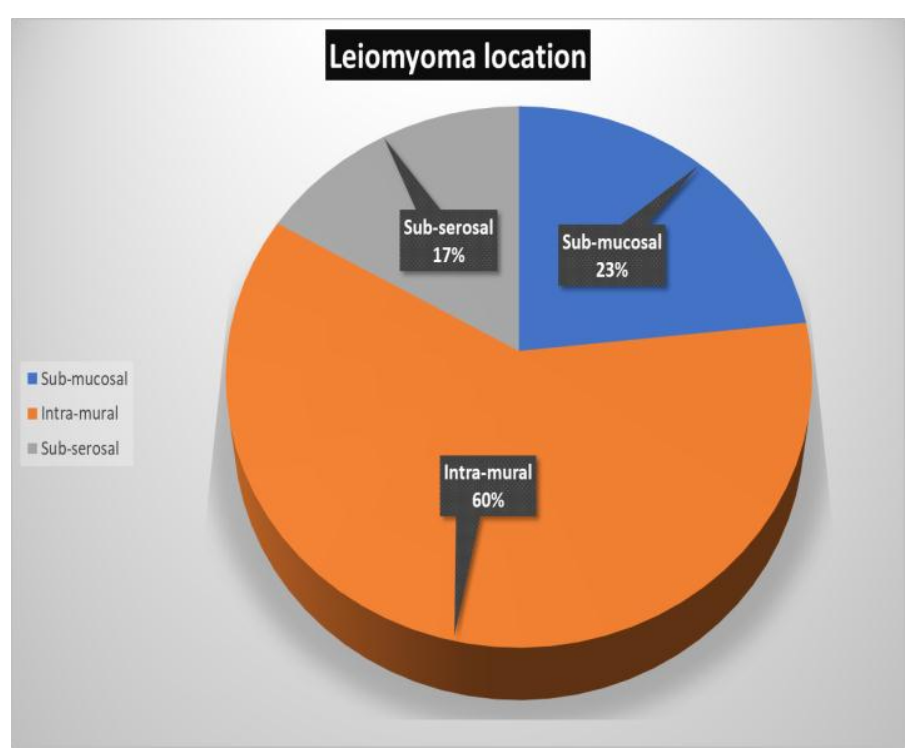

Fig. 3: Location of leiomyomas in relation to uterine layers

Recorded average uterine as well as dominant leiomyoma volumes were 770 (range: 330-1193 $\mathrm{cm}^{3}$ ), 300.5 (Range: $80-500 \mathrm{~cm}^{3}$ ) respectively (Fig. 4).

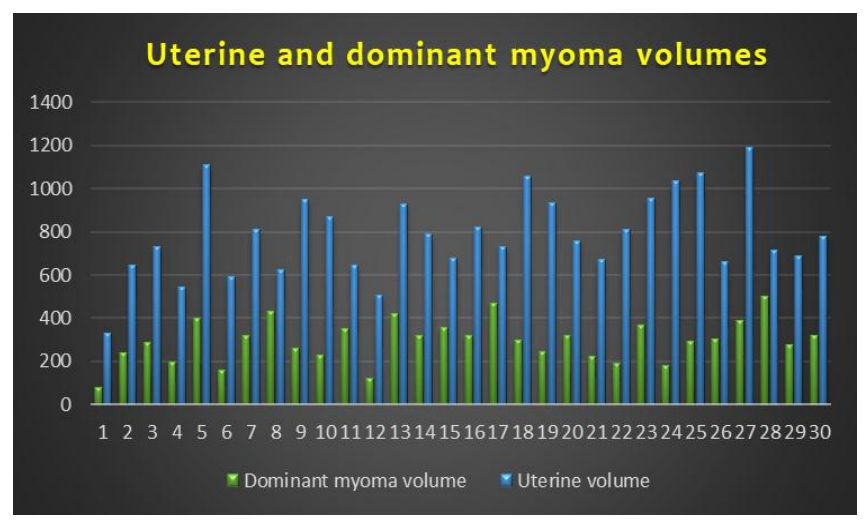

Fig. 4: uterine and dominant leiomyoma volumes as measured on preprocedural MRI

UFE procedure was technically successful on both uterine arteries in twenty-nine patients. Arterial spasm hinders bilateral uterine artery catheterization, hence unilateral approach was done in only one patient. The majority of patients were discharged at the same/ next day of the procedure with 8 (range: 6-24) hours average time of hospital stay. Overnight (> 24-hour) admission to the hospital was required in $6.7 \%$ of patients. The average number of days until patients return to normal activity post - therapy was 7 (range: 5-10) days. Minor complications were recorded in only $13.4 \%$ of patients; one having post-procedural fever treated with IM antibiotics for 5 days, another patient presented with localized hematoma managed by sufficient compression for 1 hour. Two other patients complained of persistent severe pain for which night admission was required while being administered potent analgesics and nonsteroidal anti-inflammatory agents. Neither major complication nor further gynecologic interventions occurred. Follow-up verbal questionnaires were acquired from all patients at 3,6 months. No one lost follow up visits. Most patients assured symptomatic improvement post procedural at 3 months which continued to 6 months of follow up.

Among patients presented with bleeding (n $=25)(n=24) 92,95.8 \%$ reported symptomatic improvement at three and six $M$ follow-up respectively. No significant symptomatic change between 3, 6 months follow up $(\mathrm{p}=0.255)$. Regarding patients suffered from pressure symptoms, follow up at 3 months revealed that $85 \%$ of patients had improvement (10\% significant, $75 \%$ slight), $10 \%$ had no change, $5 \%$ gave slightly worse response. While at 6 months follow up 95\% reported symptomatic improvement $(80 \%$ significant, $15 \%$ slight), $5 \%$ had no change. A highly significant time improvement between 3, 6 months follow up is notable $(\mathrm{p}=0.000)$.

While patients came complaining of pain, at $3 \mathrm{M}$ follow up $54.4 \%$ get improved, $36.4 \%$ no change, $9.1 \%$ slightly worse response meanwhile at $6 \mathrm{M}$ follow up $90.9 \%$ get improved $(36.4 \%$ significant, 54.4 slight), $9.1 \%$ stationary course of pain. There is clinical reduction of pain from 3, 6 $\mathrm{M}$ follow up visits $(\mathrm{P}=0.079$; tend to be clinically significant) (Fig. 5). Among two patients presented with infertility, one gets pregnant at 6 months follow up with no significant change between 3,6 $\mathrm{M}$ follow up ( $\mathrm{P}=0.313)$. 
Measurement of Uterine Leiomyomata Volume...

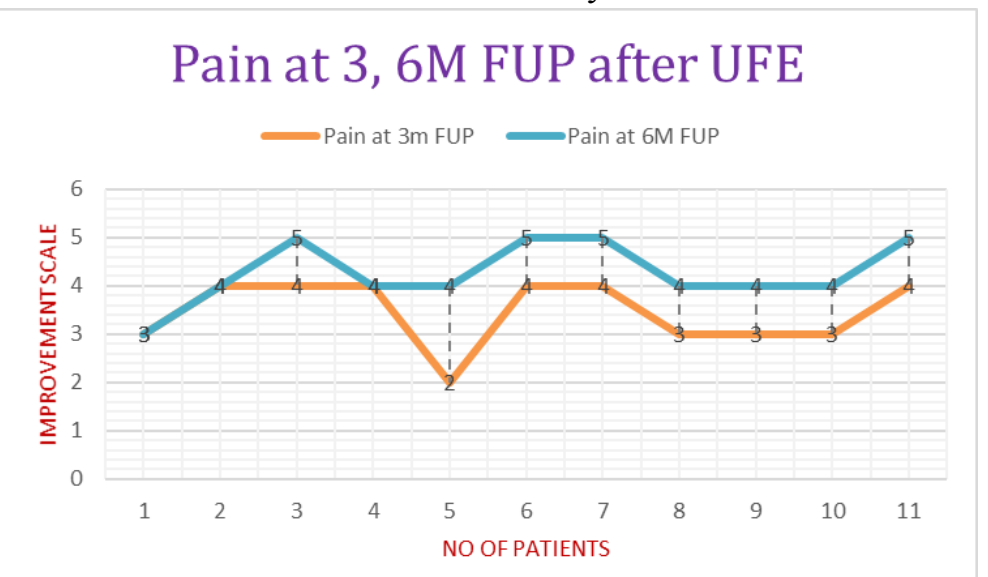

Fig. 5: pelvic pain at 3 and 6 months following uterine fibroid embolization.

Patient satisfaction paralleled symptoms change. $70 \%$ of patients were satisfied at $3 \mathrm{M}$ follow up which increased up to $86.7 \%$ at $6 \mathrm{M}$. Imaging follow-up via pelvic MRI with contrast was obtained in 30 patients at 3 , 6 months after treatment. The mean uterine volume was reduced by 25\% (standard deviation [SD] 0.03763) 3 months after treatment and was further reduced by a mean of 30\% (SD 0.02638 ) by six months after treatment. Post-procedural subtraction MRI revealed mean myoma enhancement to be $5.6+0.5 \mathrm{SI}$ in contrast to $133.4+$ 18.3 SI prior to UFE. The mean dominant fibroid volume reduced by $34 \%$ (SD 0.09247) after 3 months, $44 \%$ (SD 0.05994) after 6 months. A cumulative reduction in both uterine and dominant leiomyoma mean volume from baseline to 6 months post - UAE is evident with significant interval changes between 3, 6 months follow up (p 0.000) (Figs. 6 and 7).

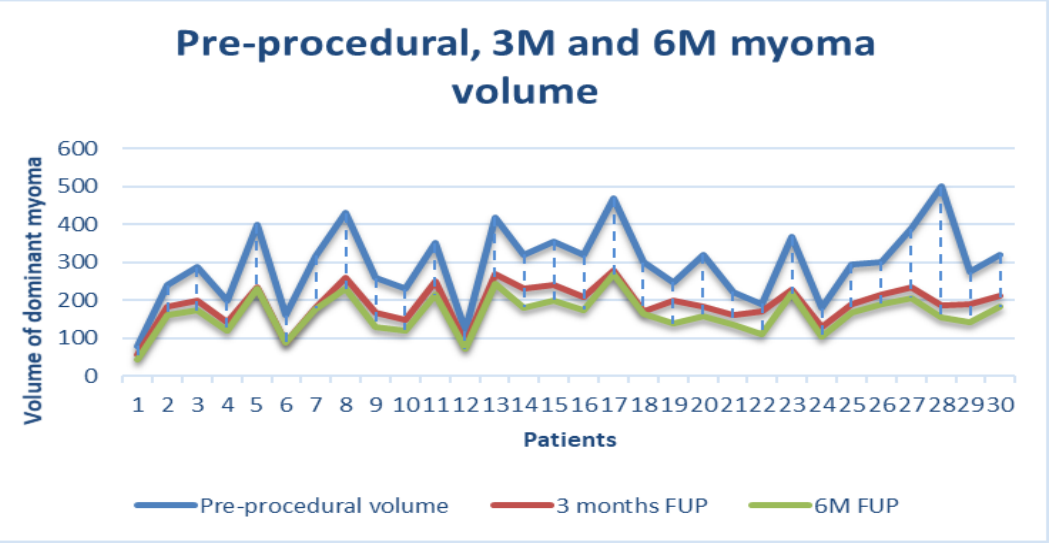

Fig. 6: pre-procedural, 3 and 6 ms leiomyoma volume as measured on MRI

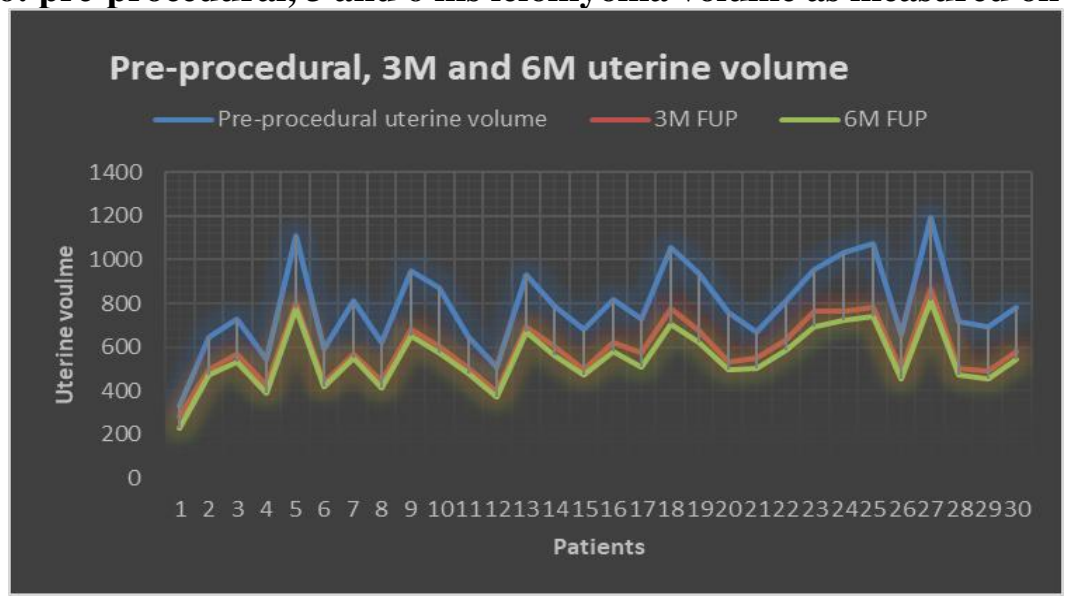

Fig. 7: pre-procedural, 3 and 6 months uterine volume as measured on MRI

Next, multiple linear regression with percent change in myoma volume as the dependent variable and patient's age, leiomyoma location whether (subserosal, submucosal or intramural) and pre-UAE myomatous volume and post-contrast enhancement as independent variables was carried-out. Only pre-UAE volume ( $\mathrm{p}=0.002)$ was the significant independent variables; where pre-procedural smaller volume was coupled with greater diminution post UFE. Conversely, pre-UAE enhancement was not an independent predictor of post-UAE volume reduction ( $\mathrm{p}=0.37)$ (Figs. 8 and 9) 


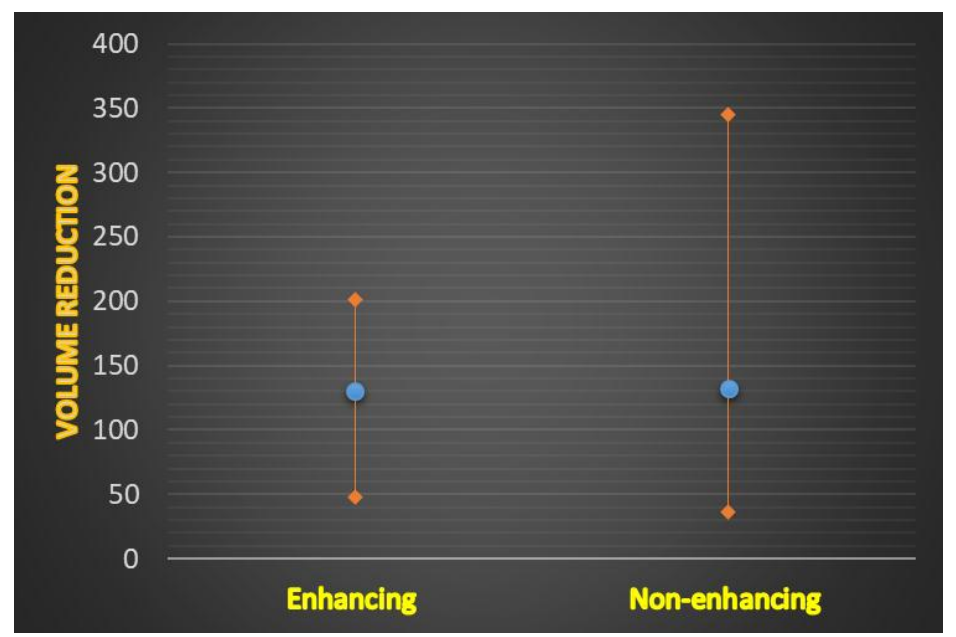

Fig. 8: Myomatous volume reduction in relation to preprocedural enhancement (Enhancement, non-enhancement).

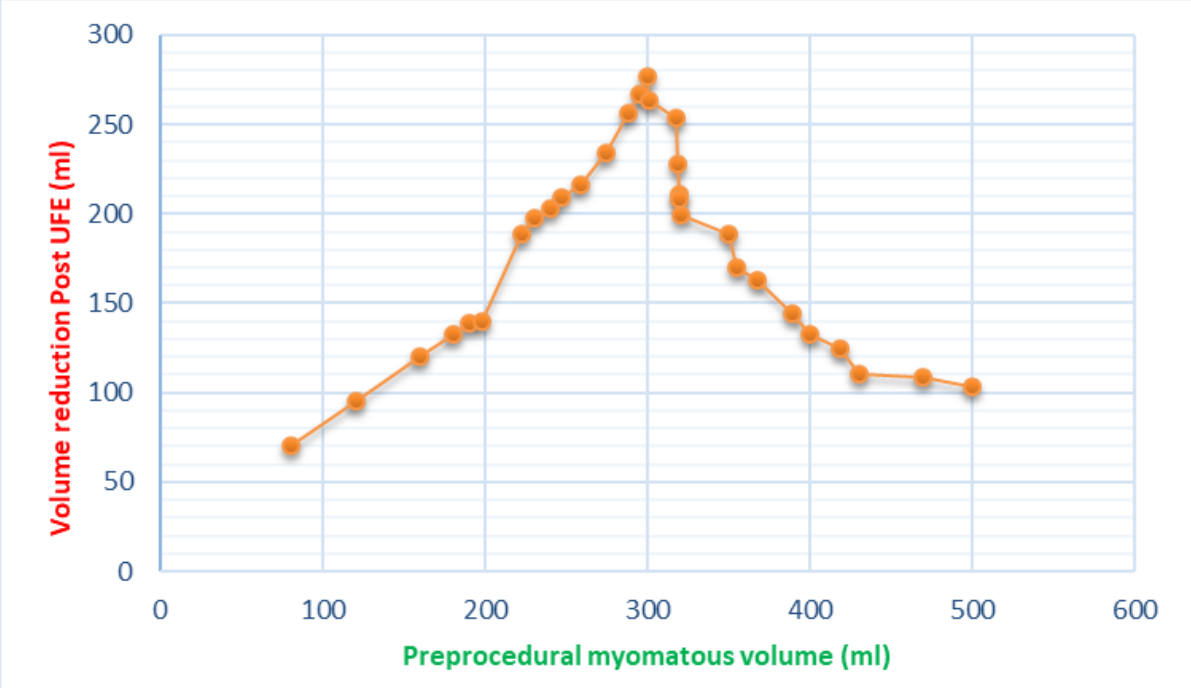

Fig. 9: Myomatous volume reduction after UFE in relation to preprocedural Myomatous volume

\section{DISCUSSION}

Liapi et al. ${ }^{(9)}$ stated that regardless of its relatively elevated cost, MR imaging is a noninvasive procedure that permits a more confident diagnosis of uterine leiomyomas while influencing patient treatment by minimizing number of unnecessary surgeries with subsequent reduction in healthcare expenditures. In concurrence with Orazio et al. ${ }^{(11)}$, precise detection and localization of leiomyomas with MR imaging, different from clinical examination and Ultrasound (US), can lead to planned therapies being changed or obviated.

Spielmann et al. ${ }^{(12)}$ within his study upon 49 patients with leiomyomas compared MR imaging and US prior to performing UFE in terms of uterine and leiomyoma volume, leiomyoma location, and total number of leiomyomas. The study found out a significant contradiction in uterine size, leiomyoma location, as well as number of leiomyomas between the two imaging modalities. Moreover, MR imaging results affected clinical management in 11 of 49 patients $(22 \%)$, where four patients were excluded from UFE, while seven women underwent embolization after being deemed unsuitable candidates for UFE at initial US evaluation. The authors stated that MR imaging provides a considerable amount of supplementary information comparable to US and affects clinical management in a substantial number of patients, making MR imaging the imaging modality of choice before UFE.

Aided by a standard phased-array surface coil, pelvic imaging with suspended respiration can be accomplished in nearly 20 minutes. Das $\boldsymbol{e t} \boldsymbol{a l}$. (13) set out that all protocols should include T2weighted images in at least two orthogonal planes (generally axial and sagittal) to define the uterine axis and determine leiomyomas location. Axial T1weighted images, opposed-phase GRE or fast spinecho (FSE), should be obtained to portray the leiomyomatous relationship to the uterus and adnexa. Moreover, they allow characterization of blood and fat signals facilitating diagnosis of concurrent uterine and adnexal disease.

In line with our protocol, three-dimensional dynamic non-enhanced and contrast-enhanced fat- 
Measurement of Uterine Leiomyomata Volume...

suppressed GRE images should be taken to determine extent of leiomyoma enhancement and define peri-uterine vessels. Moreover, this dynamic sequence can be modified to serve as MR angiography, usually in the sagittal plane to facilitate identification of parasitized ovarian vessels.

Leimyomatous number, location, size, stalk size (If pedunculated type) and contrast enhancement provide valuable prognostic information about the potential success of UFE. Therefore, they should be analyzed and reported. As proclaimed by Cura et al. ${ }^{(15)}$, it is of paramount importance to detect intracavitary submucosal or pedunculated sub-serosal leiomyomas with a narrow stalk before UFE. Initially, it was supposed that the latter is a relative contra-indication to UFE for fear of risk of separation from the uterus. The potential for leiomyomata's stalk necrosis and detachment could predispose to peritonitis, persistent pain, or infection. Nonetheless, it has been demonstrated that for pedunculated subserosal leiomyomas with a stalk diameter of $2 \mathrm{~cm}$ or larger, embolization is not coupled with increased frequency of serious complications. We encountered 2 cases of sub-serosal myomas that are pedunculated however they weren't excluded as the stalk was of $3 \mathrm{~cm}$ in diameter. Post embolization follow-up show an excellent response with no subsequent detachment or further complications. Furthermore, according to Cura et al. ${ }^{(15)}$, if there is remarkable parasitization of ovarian vasculature, consideration may be given to be embolized at the time of UFE, or perhaps at a later date if there is no significant symptom relief following UFE. We had 1 case with pelvic pain and pressure symptoms attributable to solitary huge myoma that shows auxiliary supply from ovarian vessels evident at pre-embolization angiography. Another session of embolization was planned however patient experienced symptomatic relief following first session of UFE with no indication for further interventionCurrently, there is conflicting data in the literature in regards to fibroid enhancement as a predictor of UAE response. Deshmukh et $\boldsymbol{a l} .{ }^{(14)}$ argued that UFE is not considered the most treatment choice for patients with non-enhancing symptomatic leiomyomas at pre-procedural imaging, as these de-vascularized leiomyomas are less likely to reduce in size following treatment concomitant with less symptomatic improvement. Thus, hysterectomy or myomectomy may be better options especially if the patient's complaint is likely due to bulk effect. In contrary, Deipolyi ${ }^{(16)}$ concluded that non-enhancing and presumably necrotic myomas respond to UAE, despite their relative avascularity. Though one may assume that embolization should be more effective for myomas of greater vascularity, myomas have particularly abnormal angiogenesis and vascularization patterns that are not completely understood. The myomatous avascular and hypoxic environment is paradoxically associated with more proangiogenic factors.

Williams et al. (17) specified another advantage of MR imaging that is its superiority for assessment of the adnexa and endometrium for potential disease particularly when uterus is inhabited with many leiomyomas that distort pelvic anatomy as well as exclusion of co-existent diseases as adenomyosis that has been reported in $15 \%-23 \%$ of patients with leiomyomas. Bazot et $a l$. found that sensitivity and specificity of MRI and endo-vaginal US were significantly better than those of transabdominal US for diagnosis of adenomyosis. However, MRI was more accurate than endo-vaginal US when encountered with concomitant leiomyomas. Pelage et al. (18) performed UFE for 18 women with menorrhagia cause of adenomyosis. Six months following UFE, 94\% of the women reported cessation of bleeding; however, 2 years later, nearly half of the patients experienced symptoms recurrence where additional treatment or hysterectomy was a necessity. Although UFE showed to be relatively not the most advantageous in controlling symptoms relevant to adenomyosis in contradiction to leiomyomas however it has been shown that most patients with adenomyosis have symptom relief following UFE but the rate is not as high as in those with symptoms attributable to leiomyomas. As stated by Verma et $\boldsymbol{a l} .{ }^{\left({ }^{(19)}\right.}$, routine follow-up MR imaging obtained 6 months following the procedure will ideally show complete infarction of uterine leiomyomas, evident as lack of enhancement after contrast media administration. This is sometimes coupled with T1-shortening effects of methemoglobin and variable signal intensity on T2weighted images, relying on the age of hemorrhage within the leiomyoma, a condition known as hemorrhagic infarction. Occasionally, a minute amount of gas may be noted within a leiomyoma following UFE which does not equate with a pyomyoma.

Our results with UFE procedure for symptomatic leiomyomata mirror those reported in the literature. Heavy menstrual bleeding is controlled in $85-95 \%$ of patients while $70-90 \%$ of patients document improved pressure -related manifestations. In a recent meta-analysis by Toor et al. ${ }^{(4)}$ rates of symptomatic improvement for less than 1 year follow up were 87.5 (84.7-90.0) for heavy menstrual bleeding, $86.6(82.2-90.5)$ for pressure-related symptoms, 77.5 (73.8-80.9) for 
Tamara Abdullah et al.

pelvic pain. Our results for menorrhagia, bulk related symptoms are in agreement with those, $92 \%$ of patients reporting improved menstrual bleeding and $85 \%$ of patients with pelvic pressure and other bulk-related symptoms improved at 3 months follow up post-procedural. These results remained stable with no statistical significance during the course of follow-up.

Patient satisfaction results are keeping with symptomatic improvement, $86.7 \%$ of patient gave satisfactory response at 6 months following the procedure.

Although rare, complications after UFE have been reported. MRI could serve to diagnose or exclude many post-procedure complications. Minor complications comprise local hematoma, transient pain, post-embolization syndrome as well as delivery of a leiomyoma. A hematoma following UFE is rare and manifests similarly to any other hematomas after an arterial puncture in any interventional procedure. Post-embolization syndrome is a constellation of findings that are experienced to a variable degree, comprising pelvic pain and cramping, nausea and vomiting, low-grade fever, and general malaise. Traditionally, aforementioned symptoms can be controlled conservatively with oral analgesics and acetaminophen. As Verma et al. ${ }^{(19)}$ stated, postembolization infectious complications are now seen in less than $1 \%$ of patients following UFE. Infectious complications comprise endometritis, tubo-ovarian abscess (TOA), pyomyoma, and uterine necrosis. Endometritis occurs in $0.5 \%$ of women undergoing UFE. Most patients respond well to antibiotics; yet, if resistant, hysterectomy is required. At MRI, there may be globular uterine enlargement with an intracavitary hematoma that exhibited a high signal intensity on T1-weighted images. Gas will appear as a signal void on both T1- and T2-weighted images.

Pyomyoma (aka suppurative leiomyoma) is a rare complication of UFE that occurs sequent to infarction and superimposed infection. Following UFE, patients present with sepsis and no other source of infection. Imaging findings are not pathognomonic, as gas can be seen after UFE; therefore, correlation with clinical symptoms is mandatory. Even-though uterine necrosis requiring hysterectomy takes place in less than $1 \%$ of UFE patients, contrast-enhanced MR imaging is the modality of choice for evaluating the myometrial viability and vascularity. Uterine necrosis will show no myometrial enhancement on contrastenhanced MR images.

Complications were very rare with no detectable post-procedural acute major complications which parallel meta-analysis of Toor et al. ${ }^{(4)}$. We had four minor complications; two with fever, local hematoma which were managed by antibiotics, efficient compression; two presented with intolerable pain that necessities overnight admission with potent analgesics. Pelvic pain is the most frequent reported adverse effect of UAE as a result of ischemia, hence the necessity for an appropriate pain management protocol following the procedure. Till date, no readmissions had occurred or need for subsequent gynecological interventions.

\section{CONCLUSION}

The MRI findings following UAE vary with the interval from embolization and success of the procedure. MRI with its multiplanar capabilities is typically employed to evaluate the uterus following UAE for fibroid infarction, size reduction, location change, persistent enhancement and fibroid recurrence, changes in adenomyosis, and unexpected complications that may require surgical intervention or identify women who would benefit from repeated UAE.

\section{RECOMMENDATIONS}

Based on our study, a multidisciplinary prospective study on a larger population is of paramount importance to specify precisely the clear indications, contraindications and the appropriate candidate for UAE procedure, whom shall benefit from the procedure, who will get worse.

\section{REFERENCES}

1) Vilos $G$, Allaire $C$, Laberge $P$ and Leyland $N$ (2015): The management of uterine leiomyomas. J. Obstet. Gynaecol. Can., 37(2):157-178.

2) Westcott M (2015): Uterine Fibroid Embolization. In: Urgent Interventional Therapies $.1^{\text {st }}$ ed.Wiley and Sons.London.pp:474-484.

3) Martins J, Gaudenti D, Crespo F, Ganesh D and Verma U (2016): Uncommon complication of uterine artery embolization. Case Reports in Obstetrics and Gynecology doi:10.1155/2016/8695318

4) Toor S, Jaberi A, Macdonald D, McInnes M et al. (2012): Complication rates and effectiveness of uterine artery embolization in the treatment of symptomatic leiomyomas: a systematic review and meta-analysis. AJR., 199:1153-1163.

5) Liang E, Brown B, Kirsop R, Stewart $P$ and Stuart A (2012): Efficacy of uterine artery embolization for treatment of symptomatic fibroids and adenomyosis An interim report on an Australian experience. Australian and New Zealand Journal of Obstetrics and Gynecology, 52: 106-112.

6) Maciel C, Tang Y, Sahdev A, Madureira A, Vilares-Morgado P (2016): Preprocedural MRI and MRA in planning fibroid embolization. Diagn. Interv Radio., 23(2):1-9.

7) Siddiqui $N$, Nikolaidis $P$, Hammond $N$ and Miller $F$ (2013): Uterine artery embolization: pre- and post- 
Measurement of Uterine Leiomyomata Volume...

procedural evaluation using magnetic resonance imaging. Abdom. Imaging, 38(5):1161-77.

8) Kirby J, Burrows D, Haider E, Maizlin $Z$ and Midia M (2011): Utility of MRI before and after uterine fibroid embolization: Why to do it and what to look for. Cardiovasc. Intervent. Radiol., 34:705-716.

9) Liapi E., Kamel I and Bluemke D (2005): Assessment of response of uterine fibroids and myometrium to embolization using diffusion-weighted echo-planar MR imaging. Comput .Assist. Tomogr., 29:83-86.

10) Orsini LF, Venturoli $S$, Lorusso R, Pluchinotta V, Paradisi $R$ and Bovicelli $L$ (1985): Ultrasonic findings in polycystic ovarian disease. Fertility and Sterility, 43(5):709-714.

11) Orazio T, Nicoletta $C$, Stefano $M$ et al. (2015): Role of MRI for evaluation of uterine giant myoma: case report. International Journal of Medical Imaging, 3(3): 59-62.

12) Spielmann A, Keogh C, Forster B, Martin $M$ and Machan L (2006): Comparison of MRI and sonography in the preliminary evaluation for fibroid embolization. AJR., 187:1499-1504.

13) Das R, Gonsalves M, Vlahos I, Manyonda I and Belli AM (2013): MRI assessment of uterine artery patency and fibroid infarction rates 6 months after uterine artery embolization with nonspherical polyvinyl alcohol. Cardiovascular and Interventional Radiology, 36(5): 1280-1287.

14) Deshmukh SP, Gonsalves CF, Guglielmo FF and Mitchell DG (2012): Role of MR imaging of uterine leiomyomas before and after embolization. Radiographics, 32(6):E251-81.

15) Cura M, Cura A and Bugnone A (2009): Role of Magnetic Resonance Imaging in Patient Selection for Uterine Artery Embolization. Acta Radiologica, 47:1105-1113.

16) Deipolyi A (2017): MRI enhancement predicting fibroid volume reduction to uterine artery embolization. Front Womens Healt, 2(2): 1-3.

17) Williams P, Coote J and Watkinson A (2011): PreUterine Artery Embolization MRI: Beyond Fibroids. Cardiovasc. Intervent. Radiol., 34:1143-1150.

18) Pelage JP, Jacob D, Fazel A, Namur J, Laurent A, Rymer $R$ and Le Dref $O$ (2005): Midterm results of uterine artery embolization for symptomatic adenomyosis: initial experience. Radiology, 234(3):948-953.

19) Verma S, Gonsalves C, Baltarowich O, Mitchell D et al. (2010): Spectrum of imaging findings on MRI and CT after uterine artery embolization. Abdom. Imaging, 35:118-128. 